\title{
VALIDACIÓN ALTERNATIVA \\ DEL MODELO CONTINUO ALEATORIO DE MODELACIÓN DE UN ÍNDICE DE PÉRDIDAS POR CATÁSTROFES \\ CON TASA DE DECLARACIÓN DE SINIESTROS CONSTANTE DESENCADENANTE DE LOS CAT BONDS. ESTUDIO PARA EL CASO DE INUNDACIONES EN ESPAÑA*
}

\section{AN ALTERNATIVE VALIDATION FOR MODELING LOSS-INDEX TRIGGERED CAT BONDS. A CASE STUDY OF FLOODS IN SPAIN}

\author{
MARÍA JOSÉ PÉREZ-FRUCTUOSO** \\ Fecha de recepción: 4 de septiembre de 2021 \\ Fecha de aceptación:30 de octubre de 2021 \\ Disponible en linea: 30 de diciembre de 2021
}

Para citar este artículo/To cite this article

\begin{abstract}
Perez-Fructuoso, María José, Validación alternativa del modelo continuo aleatorio de modelación de un índice de pérdidas por catástrofes con tasa de declaración de siniestros constante desencadenante de los Cat Bonds. Estudio para el caso de Inundaciones en España, 55 Rev.IberoLatinoam.Seguros, 201-220 (2021). https://doi.org/10.11144/Javeriana.ris55.vamc
\end{abstract}

doi:10.11144/Javeriana.ris55.vamc

\footnotetext{
Artículo de Investigación..

** Doctora Europea en Economía, Doctora en Ciencias Económicas y Empresariales, Licenciada en Ciencias Actuariales y Financieras. Licenciada en Ciencias Económicas y Empresariales Profesora Titular del Área de Economía Financiera y Contabilidad. Madrid Open University (UDIMA). ORCID: 0000-00023252-1631 Contacto: mariajose.perez@udima.es
} 


\section{RESUMEN}

A partir del modelo continuo de determinación de un índice de pérdidas por catástrofes para los Cat Bonds, desarrollado por PÉREZ-Fructuoso (2008 y 2009) y utilizando datos asociados a un conjunto de inundaciones ocurridas en España, se estima el parámetro fundamental del modelo propuesto, tasa instantánea de declaración de siniestros, aplicando una metodología alternativa de Mínimos Cuadrados con Restricciones y se comparan los resultados con los obtenidos utilizando la metodología tradicional de máxima verosimilitud. Adicionalmente, se obtiene la volatilidad que incorpora el proceso de Wiener en el modelo para la estimación realizada de la tasa de declaración y se verifica la bondad del ajuste mediante el cálculo de los correspondientes intervalos de confianza.

Palabras Clave: Tasa instantánea de declaración de siniestros, estimación por Mínimos Cuadrados con Restricciones, test Ji-cuadrado, Raíz del Error Cuadrático Medio, Intervalos de Confianza.

\section{ABSTRACT}

Following the continuous modelling of loss index triggers for CAT Bonds developed by PEREZ-FRUCTUOSO (2008 and 2009), this paper estimates the instantaneous loss reporting rate (the proposed model's fundamental parameter) by applying a Restricted Minimum Squares' alternative methodology to a dataset of several floods occurred in Spain. Results are compared with those stemming from the traditional methodology of maximum-likelihood, the WienerProcess-induced model's volatility is obtained, and the goodness-of-fit is verified through the calculation of the corresponding confidence intervals.

Keywords: Instantaneous claim reporting rate, Constrained least-squared Estimation, Chi-square test, Root Mean Squared Error, Confidence Intervals

SUMARIO: 1. INTRODUCCIÓN. 2. PRINCIPALES CARACTERÍSTICAS DEL MODELO DE DECLARACIÓN DE SINIESTROS CON TASA DE DECLARACIÓN DE SINIESTROS CONSTANTE. 3. VALIDACIÓN DEL MODELO PROPUESTO. 3.1. Estimación de los parámetros del modelo. 3.1.1. Estimación de la tasa de declaración de siniestros por Mínimos Cuadrados con Restricciones (MCR). 3.1.2. Estimación de la volatilidad del modelo aleatorio. 3.1.3. Intervalos de confianza para la cuantía de siniestros pendiente de declarar. 4. CONCLUSIONES. REFERENCIAS BIBLIOGRÁFICAS. 


\section{INTRODUCCIÓN}

Una de las principales formas de titulización del riesgo catastrófico son los Cat Bonds o bonos sobre catástrofes. El desencadenante de estos instrumentos puede definirse como una ratio de siniestralidad en la que se relacionan la cuantía total de las pérdidas debidas a catástrofes ocurridas durante un determinado periodo de tiempo con un valor constante, habitualmente definido como el volumen estimado de las primas destinadas a cubrir las pérdidas catastróficas consideradas. La naturaleza de esta ratio es aleatoria porque la cuantía total de las pérdidas en las que se incurre es un valor desconocido a lo largo de toda la vida del contrato: a priori ignoramos el número de catástrofes que van a producirse, su magnitud y los momentos de su ocurrencia; tampoco se conoce el ritmo de declaración de los siniestros asociados.

Partiendo del análisis empírico de la evolución de las declaraciones de siniestros después de suceder una catástrofe, Pérez-Fructuoso $(2008,2009)$ propone un modelo matemático para calcular la componente aleatoria de la ratio de siniestralidad subyacente de esta clase de activos derivados. La característica distintiva del modelo es la consideración de una nueva variable, denominada cuantía de siniestros pendiente de declarar, frente a los enfoques precedente tales como Cummins et al. (1995), Geman, et al. (1998) o NowAK et al. (2013), entre otros, que solo consideran en su sistematización la cuantía de siniestros ya declarada. De esta forma, cuando se produce una catástrofe, su volumen total es la suma de estas dos cuantías, la cuantía declarada de siniestros y la cuantía de siniestros pendiente de declarar. Sin embargo, en la modelización propuesta por PÉREZ-FruCtUOSO $(2008,2009)$ se representa la siniestralidad instantánea mediante una ecuación diferencial cierta que describe un crecimiento de la siniestralidad declarada proporcional a la cuantía de siniestros pendiente de declarar en cada momento. La evolución de esta última cuantía también viene dada por una ecuación diferencial ordinaria que simboliza una tendencia decreciente de los importes de siniestros pendientes de declarar proporcional a una tasa, de declaración de siniestros constante, denominada tasa instantánea de declaración de siniestros. Para ajustar a la realidad el modelo de evolución de las declaraciones de siniestros se incorpora la aleatoriedad perturbando esta tasa instantánea de declaración de siniestros con un ruido blanco, o diferencial del proceso de Wiener de forma que la ecuación diferencial asociada a la cuantía de siniestros pendiente de declarar se vuelve estocástica, la tasa de declaración de siniestros se convierte en un proceso estocástico con trayectorias continuas, y la cuantía de siniestros pendiente de declarar se representa a través de una tendencia constante perturbada en cada momento por la variancia que incorpora el proceso de Wiener. La cuantía declarada de siniestros resulta de la diferencia entre el volumen total de la catástrofe y la cuantía de siniestros pendiente de declarar.

En cuanto a la validación de estos modelos, la tasa de declaración de siniestros y la volatilidad del proceso de Wiener se estiman aplicando el método de Máxima Verosimilitud. Posteriormente y sobre la base de dichas estimaciones se verifica la bondad del ajuste calculando los intervalos de predicción del 90 por ciento y el 99 por ciento para el IBNR por medio de una distribución normal de dos colas, asumiendo el total de la catástrofe incurrida pérdida es igual a 100. 
En este trabajo se realiza una validación alternativa del modelo continúo desarrollado. Se propone la estimación de la tasa de declaración de siniestros constante aplicando una metodología de Mínimos Cuadrados con Restricciones lo que da lugar a valores diferentes de la tasa de declaración de siniestros. También, se determina la volatilidad que incorpora el proceso de Wiener dentro del modelo continuo aleatorio, y ello respecto a cada una de las estimaciones realizadas sobre la tasa de declaración de siniestros. Por último, se verifica la bondad de los ajustes realizados a través del estudio de la Raíz del Error Cuadrático Medio, el contraste de la Ji-Cuadrado de Pearson y el cálculo de los correspondientes intervalos de confianza.

La estructura del artículo es la siguiente. La sección 2 realiza un resumen de las principales características del modelo continuo de declaración de siniestros sobre el que se va a realizar la estimación de los parámetros. En la sección 3 propone una metodología de estimación de los parámetros del modelo por Mínimos Cuadrados con Restricciones y se comparan los resultados obtenidos con los resultantes de aplicar el método tradicional de estimación por Máxima Verosimilitud. Finalmente, la sección 4 resume los principales resultados obtenidos y concluye.

\section{PRINCIPALES CARACTERÍSTICAS DEL MODELO DE DECLARACIÓN DE SINIESTROS CON TASA DE DECLARACIÓN DE SINIESTROS CONSTANTE}

El modelo propuesto por PÉREZ-FruCtuOso (2008, 2009) calcula el índice de pérdidas desencadenante de los bonos sobre catástrofes definido como el cociente entre el total de pérdidas asociadas a una catástrofe ocurrida a lo largo de un determinado periodo de riesgo, $S(t)$, y una constante, $p$, cuyo valor depende del tipo de índice utilizado. El valor de este índice al vencimiento, $T$, viene dado por la siguiente expresión:

$$
L(T)=\frac{S(T)}{p}
$$

De forma resumida. dicho modelo supone que la cuantía de la catástrofe ocurrida en el momento $t=0, K$, es una variable aleatoria que puede definirse como la suma de dos variables, ambas referidas al momento de valoración $t$,

$$
K=S(t)+R(t)
$$

donde $S(t)$ denota la cuantía declarada de siniestros (Reported Claims, RC) y $R(t)$ la cuantía de siniestros pendiente de declarar (Incurred But Not Reported Claims, IBNRC).

A partir de la evidencia empírica se considera que, inmediatamente después de que se produce la catástrofe, la intensidad de la declaración de siniestros es elevada y decrece con el paso del tiempo hasta anularse cuando no quedan más siniestros por declarar. Este hecho se representa mediante la ecuación diferencial,

$$
d S(t)=\alpha R(t) d t
$$


en la que ${ }^{\alpha}$ es una constante denominada tasa instantánea de declaración de siniestros.

Diferenciando la ecuación (1) se obtiene:

$$
d S(t)=-d R(t)
$$

Y substituyendo este resultado en la ecuación (2) se deriva la nueva ecuación diferencial que describe la evolución de la variable $R(t)$ :

$$
d R(t)=-\alpha R(t) d t
$$

Para capturar el comportamiento irregular del proceso de declaración de siniestros, se introduce un proceso de Wiener en la ecuación (4). La irregularidad en el proceso de declaración de siniestros depende de la cuantía de siniestros pendiente de declarar. Mientras dicha cuantía es elevada, la irregularidad en las declaraciones también lo es y decrece a medida que los hace la IBNRC. Para reflejar este comportamiento, se introduce en el modelo determinista un proceso de Wiener con intensidad $\sigma R(t)$, lo cual se conoce con el nombre de movimiento Browniano geométrico. El resultado es la siguiente ecuación diferencial estocástica,

$$
d R(t)=-\alpha R(t) d t+\sigma R(t) d W_{t}
$$

con las siguientes condiciones de contorno:

- $\mathrm{Si} t=0$, entonces $R(0)=K$, en el momento en que ocurre la catástrofe, toda su cuantía está pendiente de declarar, y por tanto no hay nada declarado todavía.

- $\quad$ Si $t \rightarrow \infty$, entonces $R(t)=0$, transcurrido un tiempo lo suficientemente grande todos los daños han sido declarados y por tanto ya no quedan siniestros pendientes de declarar.

La ecuación central de este modelo es la ecuación (5). Dicha ecuación intenta reproducir el proceso de declaración de siniestros de una catástrofe donde ${ }^{\alpha}$ representa la tendencia del proceso, $\sigma$ es el valor constante que representa la volatilidad del proceso y $W_{t}$ es un proceso de Wiener estándar asociado a la catástrofe.

Una condición necesaria del modelo radica en el hecho de que $\sigma$ debe ser un valor bajo. De lo contrario, si $\sigma$ es li suficientemente grande podría producirse la circunstancia de que la tasa de declaración de siniestros fuera positiva dando lugar a un crecimiento de la cantidad de siniestros pendiente de declarar.

La solución de la ecuación (5) necesita aplicar el lema de Itô para su cálculo (Arnold, 1974), resultando,

$$
R(t)=R(0) e^{\left(-\left(\alpha+\frac{\sigma^{2}}{2}\right) t+\sigma W_{t}\right)}
$$

y teniendo en cuenta la condición de contorno inicial $R(0)=K$, la solución de la ecuación diferencial estocástica resulta:

$$
R(t)=K e^{\left(-\left(\alpha+\frac{\sigma^{2}}{2}\right) t+\sigma W_{t}\right)}
$$


La variable $R(t)$ sigue una distribución log-normal, por tanto, la variable $\ln R(t)$ seguirá una distribución normal de parámetros (JoHNSON, et al., 1994):

$$
\ln R(t) \sim N\left(\ln K-\left(\alpha+\frac{\sigma^{2}}{2}\right) t ; \sigma \sqrt{t}\right)
$$

\section{VALIDACIÓN DEL MODELO PROPUESTO}

En la aplicación práctica del modelo teórico desarrollado por PÉREZ-FrUCTUOSO (2008, 2009) para determinar un índice de pérdidas por catástrofes, los principales parámetros a estimar son la tasa de declaración de siniestros y la volatilidad incorporada por el proceso de Wiener. Para ello, se dispone de seis series temporales de datos (Tablas 1, 2, 3, 4, 5 y 6 a continuación) sobre el porcentaje declarado de siniestros y el porcentaje de la cuantía de los siniestros pendiente de declarar (PDR en tablas), ambos acumulados semanalmente, en 6 inundaciones ocurridas en distintas regiones de España entre 1991 y 2000, Alcira (01/10/1991), San Sebastián (23/06/1992), Barcelona (14/09/1999), Zaragoza (20/10/2000), Valencia (20/10/2000) y Murcia $(20 / 10 / 2000)^{1}$.

Tabla 1:

Serie de datos Alcira 01/10/1991

\begin{tabular}{|c|c|c|}
\hline & $\begin{array}{c}\text { \% Declarado } \\
\text { Real }\end{array}$ & $\begin{array}{c}\text { \% Pendiente } \\
\text { Declarar Real }\end{array}$ \\
\hline Semana & & (PDR) \\
\hline $\mathbf{0}$ & 0 & 100 \\
\hline $\mathbf{1}$ & 15.06 & 84.94 \\
\hline $\mathbf{2}$ & 46.35 & 53.65 \\
\hline $\mathbf{3}$ & 65.04 & 34.96 \\
\hline $\mathbf{4}$ & 75.95 & 24.05 \\
\hline $\mathbf{5}$ & 81.14 & 18.86 \\
\hline $\mathbf{6}$ & 86.64 & 13.36 \\
\hline $\mathbf{7}$ & 89.47 & 10.53 \\
\hline $\mathbf{8}$ & 91.96 & 8.04 \\
\hline $\mathbf{9}$ & 93.06 & 6.94 \\
\hline $\mathbf{1 0}$ & 94.77 & 5.23 \\
\hline $\mathbf{1 1}$ & 95.92 & 4.08 \\
\hline $\mathbf{1 2}$ & 96.29 & 3.71 \\
\hline $\mathbf{1 3}$ & 96.44 & 3.56 \\
\hline $\mathbf{1 4}$ & 97.4 & 2.6 \\
\hline
\end{tabular}

Tabla 2:

Serie de datos San Sebastián 23/06/1992

\begin{tabular}{|c|c|c|}
\hline Periodo & $\begin{array}{c}\text { \% Declarado } \\
\text { Real }\end{array}$ & $\begin{array}{c}\text { \% Pendiente } \\
\text { Declarar Real }\end{array}$ \\
\hline Semana & & (PDR) \\
\hline $\mathbf{0}$ & 0 & 100 \\
\hline $\mathbf{1}$ & 11.92 & 88.08 \\
\hline $\mathbf{2}$ & 63.96 & 36.04 \\
\hline $\mathbf{3}$ & 76.33 & 23.67 \\
\hline $\mathbf{4}$ & 83.32 & 16.68 \\
\hline $\mathbf{5}$ & 87.71 & 12.29 \\
\hline $\mathbf{6}$ & 90.06 & 9.94 \\
\hline $\mathbf{7}$ & 91.28 & 8.72 \\
\hline $\mathbf{8}$ & 92.24 & 7.76 \\
\hline $\mathbf{9}$ & 93.2 & 6.8 \\
\hline $\mathbf{1 0}$ & 94.22 & 5.78 \\
\hline $\mathbf{1 1}$ & 94.82 & 5.18 \\
\hline $\mathbf{1 2}$ & 95.67 & 4.33 \\
\hline $\mathbf{1 3}$ & 96.55 & 3.45 \\
\hline $\mathbf{1 4}$ & 97.31 & 2.69 \\
\hline
\end{tabular}

\footnotetext{
Estos datos fueron elaborados de forma expresa por el Consorcio de Compensación de Seguros (dependiente del Ministerio de Economía) para realizar un estudio determinado. La forma en que están disponibles los datos, en porcentaje sobre la cuantía total declarada semanalmente, hace que no afecte el paso del tiempo en los mismos y permite evitar la brecha temporal para utilizarlos en diferentes estudios a lo largo del tiempo. La cuantía de la catástrofe analizada podría ser mayor en la actualidad, pero el porcentaje declarado en la primera semana seguiría siendo aproximadamente el mismo (fundamentalmente porque en la mayoría de los casos, la población, las infraestructuras públicas y las viviendas no se han visto prácticamente modificadas en las zonas afectadas, después de la reconstrucción).
} 


\begin{tabular}{|c|c|c|}
\hline & $\begin{array}{c}\text { \% Declarado } \\
\text { Real }\end{array}$ & $\begin{array}{c}\text { \% Pendiente } \\
\text { Declarar Real }\end{array}$ \\
\hline Semana & & (PDR) \\
\hline $\mathbf{1 5}$ & 98.25 & 1.75 \\
\hline $\mathbf{1 6}$ & 98.7 & 1.3 \\
\hline $\mathbf{1 7}$ & 99.23 & 0.77 \\
\hline $\mathbf{1 8}$ & 99.71 & Periodo \\
\hline $\mathbf{1 9}$ & 100 & 0.0 \\
\hline
\end{tabular}

\begin{tabular}{|c|c|c|}
\hline Periodo & $\begin{array}{c}\text { \% Declarado } \\
\text { Real }\end{array}$ & $\begin{array}{c}\text { \% Pendiente } \\
\text { Declarar Real }\end{array}$ \\
\hline Semana & & (PDR) \\
\hline $\mathbf{1 5}$ & 98.19 & 1.81 \\
\hline $\mathbf{1 6}$ & 98.41 & 1.59 \\
\hline $\mathbf{1 7}$ & 98.61 & 1.39 \\
\hline $\mathbf{1 8}$ & 98.84 & 1.16 \\
\hline $\mathbf{1 9}$ & 99.04 & 0.96 \\
\hline $\mathbf{2 0}$ & 99.24 & 0.76 \\
\hline $\mathbf{2 1}$ & 99.55 & 0.45 \\
\hline $\mathbf{2 2}$ & 99.72 & 0.28 \\
\hline $\mathbf{2 3}$ & 99.8 & 0.2 \\
\hline $\mathbf{2 4}$ & 99.83 & 0.17 \\
\hline $\mathbf{2 5}$ & 99.89 & 0.11 \\
\hline $\mathbf{2 6}$ & 99.94 & 0.06 \\
\hline $\mathbf{2 7}$ & 100 & 0.0 \\
\hline
\end{tabular}

Tabla 3:

Serie de datos Barcelona (14/09/1999)

\begin{tabular}{|c|c|c|}
\hline Periodo & $\begin{array}{c}\text { \% Declarado } \\
\text { Real }\end{array}$ & $\begin{array}{c}\text { \% Pendiente } \\
\text { Declarar Real }\end{array}$ \\
\hline Semana & & (PDR) \\
\hline $\mathbf{0}$ & 0 & 100 \\
\hline $\mathbf{1}$ & 9.319899244 & 90.68010076 \\
\hline $\mathbf{2}$ & 31.62354019 & 68.37645981 \\
\hline $\mathbf{3}$ & 49.32447905 & 50.67552095 \\
\hline $\mathbf{4}$ & 58.57568125 & 41.42431875 \\
\hline $\mathbf{5}$ & 68.42225784 & 31.57774216 \\
\hline $\mathbf{6}$ & 74.57064346 & 25.42935654 \\
\hline $\mathbf{7}$ & 80.4442409 & 19.5557591 \\
\hline $\mathbf{8}$ & 83.31806732 & 16.68193268 \\
\hline $\mathbf{9}$ & 86.7185711 & 13.2814289 \\
\hline $\mathbf{1 0}$ & 89.45500343 & 10.54499657 \\
\hline $\mathbf{1 1}$ & 91.84795054 & 8.152049462 \\
\hline $\mathbf{1 2}$ & 93.19899244 & 6.801007557 \\
\hline $\mathbf{1 3}$ & 93.8745134 & 6.125486604 \\
\hline $\mathbf{1 4}$ & 96.58804671 & 3.411953286 \\
\hline $\mathbf{1 5}$ & 96.58804671 & 3.411953286 \\
\hline $\mathbf{1 6}$ & 97.38951225 & 2.610487749 \\
\hline $\mathbf{1 7}$ & 98.19097779 & 1.809022212 \\
\hline $\mathbf{1 8}$ & 98.74055416 & 1.259445844 \\
\hline $\mathbf{1 9}$ & 99.43897412 & 0.561025876 \\
\hline $\mathbf{2 0}$ & 100 & 0.0 \\
\hline & & \\
\hline
\end{tabular}

Tabla 4:

Serie de datos Zaragoza (20/10/2000)

\begin{tabular}{|c|c|c|}
\hline Periodo & $\begin{array}{c}\text { \% Declarado } \\
\text { Real }\end{array}$ & $\begin{array}{c}\text { \% Pendiente } \\
\text { Declarar Real }\end{array}$ \\
\hline Semana & & (PDR) \\
\hline $\mathbf{0}$ & 0 & 100 \\
\hline $\mathbf{1}$ & 14.05082212 & 85.94917788 \\
\hline $\mathbf{2}$ & 38.26606876 & 61.73393124 \\
\hline $\mathbf{3}$ & 65.02242152 & 34.97757848 \\
\hline $\mathbf{4}$ & 75.93423019 & 24.06576981 \\
\hline $\mathbf{5}$ & 85.94917788 & 14.05082212 \\
\hline $\mathbf{6}$ & 87.59342302 & 12.40657698 \\
\hline $\mathbf{7}$ & 91.47982063 & 8.520179372 \\
\hline $\mathbf{8}$ & 94.76831091 & 5.231689088 \\
\hline $\mathbf{9}$ & 94.76831091 & 5.231689088 \\
\hline $\mathbf{1 0}$ & 94.91778774 & 5.082212257 \\
\hline $\mathbf{1 1}$ & 96.56203288 & 3.437967115 \\
\hline $\mathbf{1 2}$ & 96.41255605 & 3.587443946 \\
\hline $\mathbf{1 3}$ & 97.75784753 & 2.242152466 \\
\hline $\mathbf{1 4}$ & 98.80418535 & 1.195814649 \\
\hline $\mathbf{1 5}$ & 99.40209268 & 0.597907324 \\
\hline $\mathbf{1 6}$ & 99.40209268 & 0.597907324 \\
\hline $\mathbf{1 7}$ & 99.40209268 & 0.597907324 \\
\hline $\mathbf{1 8}$ & 99.55156951 & 0.448430493 \\
\hline $\mathbf{1 9}$ & 99.55156951 & 0.448430493 \\
\hline $\mathbf{2 0}$ & 99.55156951 & 0.448430493 \\
\hline $\mathbf{2 1}$ & 99.55156951 & 0.448430493 \\
\hline $\mathbf{2 2}$ & 100 & 0.0 \\
\hline & & \\
\hline
\end{tabular}


Tabla 5:

Serie de datos Valencia (20/10/2000)

\begin{tabular}{|c|c|c|}
\hline Periodo & $\begin{array}{c}\text { \% Declarado } \\
\text { Real }\end{array}$ & $\begin{array}{c}\text { \% Pendiente } \\
\text { Declarar Real }\end{array}$ \\
\hline Semana & 0 & (PDR) \\
\hline $\mathbf{0}$ & 100 \\
\hline $\mathbf{1}$ & 2.459480902 & 97.5405191 \\
\hline $\mathbf{2}$ & 19.82318939 & 80.17681061 \\
\hline $\mathbf{3}$ & 39.85039102 & 60.14960898 \\
\hline $\mathbf{4}$ & 56.84007707 & 43.15992293 \\
\hline $\mathbf{5}$ & 68.03808228 & 31.96191772 \\
\hline $\mathbf{6}$ & 72.44701349 & 27.55298651 \\
\hline $\mathbf{7}$ & 80.46016094 & 19.53983906 \\
\hline $\mathbf{8}$ & 84.71041596 & 15.28958404 \\
\hline $\mathbf{9}$ & 85.24311459 & 14.75688541 \\
\hline $\mathbf{1 0}$ & 85.29978465 & 14.70021535 \\
\hline $\mathbf{1 1}$ & 88.93800295 & 11.06199705 \\
\hline $\mathbf{1 2}$ & 91.54482602 & 8.455173977 \\
\hline $\mathbf{1 3}$ & 93.01824776 & 6.981752238 \\
\hline $\mathbf{1 4}$ & 93.78896067 & 6.211039329 \\
\hline $\mathbf{1 5}$ & 94.8316899 & 5.168310099 \\
\hline $\mathbf{1 6}$ & 95.78374702 & 4.216252975 \\
\hline $\mathbf{1 7}$ & 96.49778987 & 3.502210133 \\
\hline $\mathbf{1 8}$ & 97.27983679 & 2.72016321 \\
\hline $\mathbf{1 9}$ & 97.74453134 & 2.255468661 \\
\hline $\mathbf{2 0}$ & 98.11855378 & 1.88144622 \\
\hline $\mathbf{2 1}$ & 98.31123201 & 1.688767993 \\
\hline $\mathbf{2 2}$ & 98.3905701 & 1.609429899 \\
\hline $\mathbf{2 3}$ & 99.10461294 & 0.895387057 \\
\hline $\mathbf{2 4}$ & 99.45596736 & 0.544032642 \\
\hline $\mathbf{2 5}$ & 99.63731157 & 0.362688428 \\
\hline $\mathbf{2 6}$ & 99.80732177 & 0.192678227 \\
\hline $\mathbf{2 7}$ & 100 & 0.0 \\
\hline & & \\
\hline
\end{tabular}

Tabla 6:

Serie de datos Murcia (20/10/2000)

\begin{tabular}{|c|c|c|}
\hline Periodo & $\begin{array}{c}\text { \% Declarado } \\
\text { Real }\end{array}$ & $\begin{array}{c}\text { \% Pendiente } \\
\text { Declarar Real }\end{array}$ \\
\hline Semana & 0 & (PDR) \\
\hline $\mathbf{0}$ & 11.54294598 & 88.45705402 \\
\hline $\mathbf{1}$ & 24.44617178 & 75.55382822 \\
\hline $\mathbf{2}$ & 51.30198212 & 48.69801788 \\
\hline $\mathbf{3}$ & 68.86902449 & 31.13097551 \\
\hline $\mathbf{4}$ & 78.58530898 & 21.41469102 \\
\hline $\mathbf{5}$ & 84.22075398 & 15.77924602 \\
\hline $\mathbf{6}$ & 88.72910999 & 11.27089001 \\
\hline $\mathbf{7}$ & 91.29420909 & 8.705790906 \\
\hline $\mathbf{8}$ & 91.76059075 & 8.23940925 \\
\hline $\mathbf{9}$ & 93.43179168 & 6.568208317 \\
\hline $\mathbf{1 0}$ & 94.63661096 & 5.36338904 \\
\hline $\mathbf{1 1}$ & 95.99689079 & 4.003109211 \\
\hline $\mathbf{1 2}$ & 96.618733 & 3.381267003 \\
\hline $\mathbf{1 3}$ & 97.39603576 & 2.603964244 \\
\hline $\mathbf{1 4}$ & 97.20171007 & 2.798289934 \\
\hline $\mathbf{1 5}$ & 97.70695686 & 2.29304314 \\
\hline $\mathbf{1 6}$ & 97.745822 & 2.254178002 \\
\hline $\mathbf{1 7}$ & 97.86241741 & 2.137582588 \\
\hline $\mathbf{1 8}$ & 98.32879907 & 1.671200933 \\
\hline $\mathbf{1 9}$ & 98.71745045 & 1.282549553 \\
\hline $\mathbf{2 0}$ & 98.91177614 & 1.088223863 \\
\hline $\mathbf{2 1}$ & 99.06723669 & 0.932763311 \\
\hline $\mathbf{2 2}$ & 99.33929265 & 0.660707346 \\
\hline $\mathbf{2 3}$ & 99.37815779 & 0.621842208 \\
\hline $\mathbf{2 4}$ & 99.33929265 & 0.660707346 \\
\hline $\mathbf{2 5}$ & 99.84453945 & 0.155460552 \\
\hline $\mathbf{2 6}$ & 100 & 0.0 \\
\hline $\mathbf{2 7}$ & & \\
\hline & & \\
\hline
\end{tabular}

\subsection{Estimación de los parámetros del modelo}

En este trabajo, la estimación de la tasa de declaración de siniestros se ha realizado aplicando una metodología de Mínimos Cuadrados con Restricciones sobre el modelo determinista resultante de calcular la esperanza matemática del modelo aleatorio con tasa de declaración de siniestros constante (PÉREZ-Fructuoso et al., 2003). Los valores obtenidos aplicando dicha metodología se comparan con los resultantes de utilizar el método tradicional de Máxima Verosimilitud (PÉrez-Fructuoso, 2008, 2009) a través de la Raíz Cuadrada del Error Cuadrático Medio. A partir de los datos disponibles, también se desarrolla una metodología de Mínimos Cuadros para estimar la volatilidad del proceso de Wiener y se verifica la bondad del ajuste determinando los correspondientes intervalos de predicción. 


\subsubsection{Estimación de la tasa de declaración de siniestros por Minimos Cuadrados con Restricciones (MCR)}

Utilizando una metodología de Mínimos Cuadrados con Restricciones (MCR), la tasa de declaración de siniestros estimada es el mínimo valor de $\alpha^{\wedge}$ tal que verifica el siguiente problema de optimización (VAN DEN BoOs, 2007),

$$
\begin{aligned}
& \min \left(\sum_{t=1}^{n}\left(P D R_{t}-e^{-\hat{\alpha} t}\right)^{2}\right) \\
& \text { sujeto a } \sum_{t=1}^{n} \frac{P D R_{t}}{e^{-\hat{\alpha} t}}=n
\end{aligned}
$$

donde $P D R_{t}$ es la cuantía de siniestros pendiente de declarar real semanal $\mathrm{y}^{e^{-\hat{\alpha} \cdot(t)}}$ es la cuantía de siniestros pendiente declarar esperada cada semana.

Utilizando este procedimiento, la cuantía de siniestros pendiente de declarar estimada semanal, $P D E_{\text {t, }}$ se obtiene como sigue:

$$
P D E_{t}=e^{-\widehat{\alpha} \cdot t}
$$

Los valores de la tasa de declaración de siniestros derivados de aplicar esta metodología de estimación, y los obtenidos utilizando la metodología tradicional de Máxima Verosimilitud (MV), se presentan en la siguiente tabla resumen (Tabla 7):

Tabla 7: Estimación MCR y MV de la tasa de declaración de siniestros

\begin{tabular}{|c|c|c|}
\hline Serie & Tasa Estimada MCR, & Tasa Estimada MV, \\
\hline Alcira & 0.288209 & 0.3035086722 \\
San Sebastián & 0.2742304 & 0.2677497687 \\
Barcelona & 0.2309302 & 0.2573436251 \\
Zaragoza & 0.3024744 & 0.2080287412 \\
Valencia & 0.1729236 & 0.2281115136 \\
Murcia & 0.230796 & 0.2132287838 \\
\hline
\end{tabular}

Los Gráficos 1, 2, 3, 4, 5 y 6 a continuación muestran de forma visual la bondad de los ajustes sobre declaraciones pendientes reales y estimadas utilizando la tasa estimada por MCR en las series de datos utilizadas:

\section{Gráfico 1: Alcira}

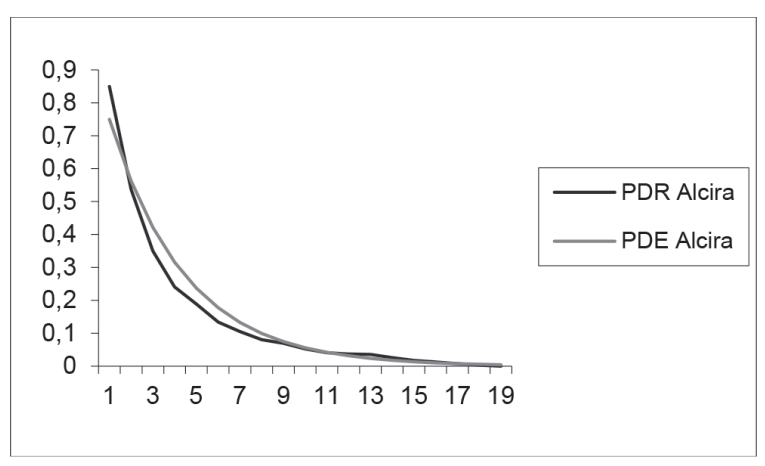




\section{Gráfico 2: San Sebastián}

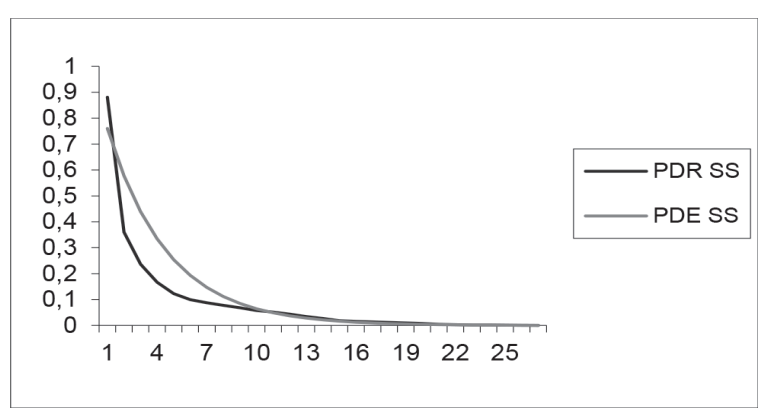

\section{Gráfico 3: Barcelona}

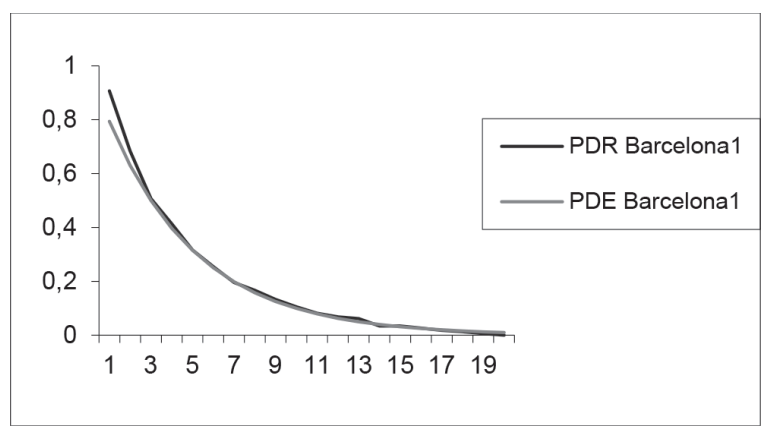

Gráfico 4: Zaragoza

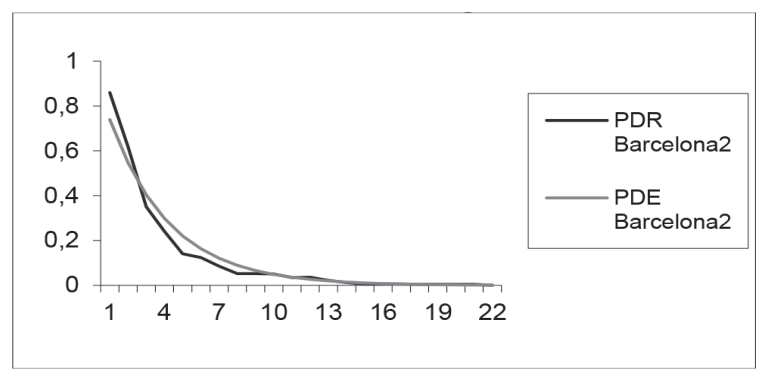

Gráfico 8: Valencia

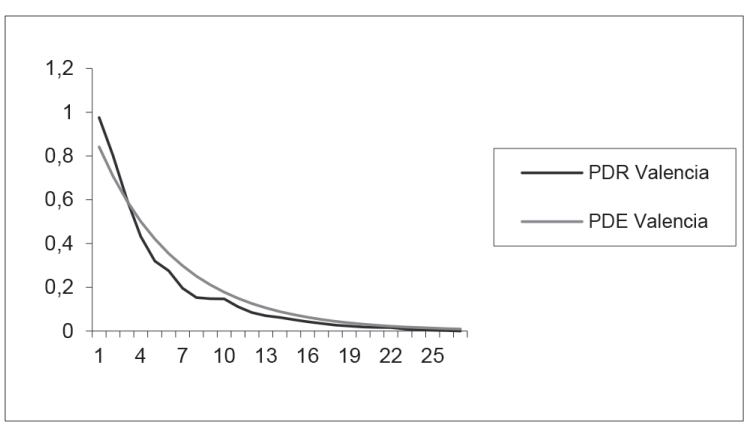




\section{Gráfico 9: Murcia}

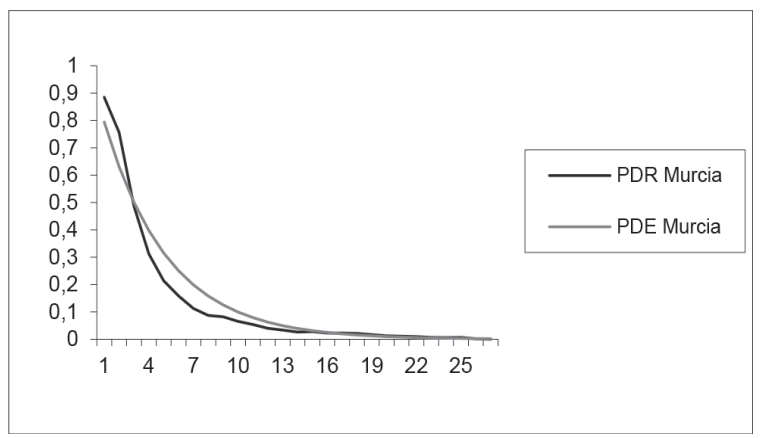

A continuación, se calcula la Raíz Cuadrada del Error Cuadrático Medio (RECM) ${ }^{2}$ existente entre los valores reales y los estimados y se comparan dichos valores con los obtenidos en los ajustes realizados mediante la estimación de la tasa de declaración de siniestros obtenida por el método de Máxima Verosimilitud. Los resultados de los diferentes valores del RECM se muestran en la Tabla 8 siguiente:

Tabla 8: RECM para tasa de declaración de siniestros MCR y MV

\begin{tabular}{|c|c|c|}
\hline Serie & RECM tasa estimada MCR & RECM tasa estimada MV \\
\hline Alcira & 3,776992346 & 3.2541320138 \\
San Sebastián & 7,743016531 & 7.9264064062564 \\
Barcelona 1 & 2,877476674 & 4.3877716557 \\
Zaragoza & 4,07856948 & 8.0230716144 \\
Valencia & 5,477043637 & 5.2483780645 \\
Murcia & 4,996582842 & 5.8600227682 \\
\hline Media & 4,824946918 & 5,7832970622 \\
\hline
\end{tabular}

Resulta evidente que la media de la raíz de los errores es menor cuando el ajuste se realiza con la tasa de declaración de siniestros estimada por MCR. En los casos de Barcelona 1, Zaragoza y Murcia además la raíz del error cuando la tasa es la estimada por MCR es significativamente menor que cuando la tasa se estima por MV.

Para verificar la bondad del ajuste realizado por MCR utilizamos la prueba $\chi^{2}$ de Pearson que permite averiguar si la distribución empírica de los datos se ajusta a una determinada distribución teórica.

En el caso del test de bondad de ajuste ji-cuadrado las hipótesis nula, $H_{0}$, y alternativa, $H_{1, \text { son: }}$

2 La raíz cuadrada del Error Cuadrático Medio (RECM) se define la raíz cuadrada de las sumas al cuadrado de las diferencias entre los valores estimados y los valores reales, en este caso de la cuantía de siniestros pendiente de declarar:

$$
R E C M=\sqrt{\frac{1}{n} \sum_{i=1}^{n}\left(\widehat{P D E}_{i}-D P R_{i}\right)^{2}}
$$


- $H_{0}$ : Los datos originales y los estimados proceden de la misma distribución.

- $H_{1}$ : Los datos originales y los estimados tienen distribuciones distintas.

Para calcular el estadístico de prueba del contraste calculamos,

$$
\chi_{\text {prueba }}^{2}=\sum_{i=1}^{k} \frac{\left(O_{i}-E_{i}\right)^{2}}{E_{i}}
$$

donde $O_{i}$ es el valor observado ${ }^{i}$-ésimo, $E_{i}$ es el valor esperado ${ }^{i}$-ésimo y $\mathrm{k}$ son el número de observaciones o clases analizadas.

Entonces, si el valor del estadístico de prueba es mayor que el valor crítico de la distribución ji-cuadrado con $k-1$ grados de libertad para un nivel de significación del $\alpha \%$ se rechaza la hipótesis nula. En caso contrario, no se rechaza. Si medimos la validez del contraste a través del $P$-valor, si el $P$-valor es menor que el nivel de significación establecido en dicho contraste se rechaza la hipótesis nula, mientras que si es mayor se acepta.

Los resultados del contraste para la serie de datos analizada y para un nivel de significación del 5\% son los siguientes (Tabla 9):

Tabla 9: Contraste Ji-Cuadrado de bondad de ajuste

\begin{tabular}{|c|c|c|c|}
\hline & Estadístico de prueba & Valor crítico en tablas & p-valor \\
\hline Alcira & 9.409182281 & 28.8693 & 0.949497086 \\
San Sebastián & 44.64977764 & 38.8851 & 0.012855904 \\
Barcelona & 4.161848638 & 30.1435 & 0.999855733 \\
Zaragoza & 12.84142821 & 33.9245 & 0.914076486 \\
Valencia & 30.56367149 & 38.8851 & 0.244919958 \\
Murcia & 25.81808575 & 38.8851 & 0.473138634 \\
\hline
\end{tabular}

Como se observa en los resultados obtenidos, en todas las series consideradas, menos en la serie de San Sebastián, no podemos rechazar la hipótesis de que los datos originales y los estimados proceden de la misma distribución. En este caso, podemos afirmar que la cuantía de siniestros pendiente de declarar $R(t)$ sigue una distribución log-normal y su logaritmo neperiano, $\ln R(t)$, sigue una distribución normal. En el caso de la inundación de San Sebastián el contraste permite aceptar la hipótesis nula para un nivel de significación del 1\%.

\subsubsection{Estimación de la volatilidad del modelo aleatorio}

El modelo original dado por la ecuación (6), la variable aleatoria cuantía de los siniestros pendiente de declarar se distribuye log-normalmente y su esperanza matemática coincide con la cuantía de siniestros pendiente de declarar de un modelo exponencial determinista: 


$$
E(R(t))=K e^{-\alpha t}
$$

Entonces, $\ln R(t)$ es el proceso gaussiano,

$$
\ln R(t) \sim \mathrm{N}\left(\ln K-\left(\alpha+\frac{\sigma^{2}}{2}\right) t ; \sigma \sqrt{t}\right)
$$

Sin embargo, es posible obtener una expresión mejor para estimar los parámetros $\alpha_{\mathrm{y}}$ $\sigma$ realizando el siguiente cálculo:

$$
\frac{R(t)}{R(t-1)}=\frac{K e^{-\left(\alpha+\frac{\sigma^{2}}{2}\right) t+\sigma W_{t}}}{K e^{-\left(\alpha+\frac{\sigma^{2}}{2}\right)(t-1)+\sigma W_{t-1}}}=e^{-\left(\alpha+\frac{\sigma^{2}}{2}\right)+\sigma W_{1}}
$$

Esto significa que $\frac{R(t)}{R(t-1)}$ sigue una distribución log-normal siendo los parámetros de la distribución normal asociada,

$$
\ln \frac{R(t)}{R(t-1)} \sim N\left(-\left(\alpha+\frac{\sigma^{2}}{2}\right) ; \sigma\right)
$$

o bien:

$$
-\ln \frac{R(t)}{D(t-1)}-\alpha \sim N\left(\frac{\sigma^{2}}{2}\right.
$$

A partir de los resultados obtenidos, si simbolizamos como $\bar{X}_{\text {y }} \bar{S}_{X}^{2}$ respectivamente, a la media y variancia muestral de la distribución normal asociada al logaritmo de las variaciones semanales de la cuantía de siniestros pendiente de declarar, y llamamos $\hat{\sigma}^{2}$ al estimador de la variancia muestral, el valor de este estimador será el que resulte de minimizar la siguiente suma de errores al cuadrado,

$$
\min \left(\left(\bar{X}-\frac{\hat{\sigma}^{2}}{2}\right)^{2}+\left(\bar{S}_{X}^{2}-\hat{\sigma}^{2}\right)^{2}\right)
$$

de donde se obtiene:

$$
\hat{\sigma}^{2}=\frac{2}{5}\left(\bar{X}+2 \bar{S}_{X}^{2}\right)
$$

Aplicando el estimador resultante a las series de datos disponibles y para los valores de la tasa de declaración de siniestros estimada por MCR, los valores estimados para la volatilidad del proceso estocástico (desviación estimada) definido para la cuantía de siniestros pendiente de declarar, se muestran en la siguiente tabla resumen:

Tabla 10: Estimación de la volatilidad para MCR

\begin{tabular}{|c|c|c|}
\hline Serie & Variancia Estimada & Desviación estimada \\
\hline Alcira & 0.046453703 & 0.215531212 \\
San Sebastián & 0.031486417 & 0.177444124 \\
Barcelona & 0.040721656 & 0.201796075 \\
Zaragoza & 0.028540072 & 0.168938072 \\
Valencia & 0.046575574 & 0.215813749 \\
Murcia & 0.067622054 & 0.260042409 \\
\hline
\end{tabular}




\subsubsection{Intervalos de confianza para la cuantia de siniestros pendiente de declarar}

A continuación, precisamos la bondad del ajuste realizado para la tasa instantánea de declaración de siniestros y la volatilidad del proceso de Wiener, calculando los intervalos de predicción del 90\% y del 99\% y, por tanto, para un nivel de significación del $10 \%$ y del $1 \%$, en una normal con 2 colas, para la distribución normal que sigue el logaritmo de las variaciones de la cuantía de siniestros pendiente de declarar.

Los intervalos de predicción para las series de datos disponibles se muestran en las Tablas 11, 12, 13, 14, 15 y 16 y los Gráficos 10, 11, 12, 13, 14 y 15 siguientes:

Tabla 11: Intervalos de predicción Serie de datos Alcira

\begin{tabular}{|c|c|c|c|}
\hline $\begin{array}{c}\text { Intervalo Inferior } \\
\mathbf{9 0 \%}\end{array}$ & $\begin{array}{c}\text { Intervalo Superior } \\
\mathbf{9 0 \%}\end{array}$ & $\begin{array}{c}\text { Intervalo Inferior } \\
\mathbf{9 9 \%}\end{array}$ & $\begin{array}{c}\text { Intervalo Superior } \\
\mathbf{9 9 \%}\end{array}$ \\
\hline 51.37835824 & 104.4022921 & 42.0375271 & 127.6007115 \\
32.49009631 & 88.55834949 & 24.46313422 & 117.6165441 \\
21.25985731 & 72.59562077 & 15.01841614 & 102.7653332 \\
14.15959076 & 58.46693446 & 9.479044822 & 87.33663363 \\
9.537831501 & 46.55878469 & 6.08964302 & 72.92214696 \\
6.476424573 & 36.77953427 & 3.961678921 & 60.12599312 \\
4.424488251 & 28.87810875 & 2.601981323 & 49.10521522 \\
3.037263772 & 22.56521834 & 1.721887244 & 39.80314064 \\
2.093203424 & 17.56308769 & 1.146521623 & 32.06491231 \\
1.447343117 & 13.62482906 & 0.767363152 & 25.6981359 \\
1.003581361 & 10.5399908 & 0.515859872 & 20.50506133 \\
0.697575886 & 8.133764901 & 0.348112034 & 16.29911553 \\
0.48591276 & 6.263470367 & 0.235699969 & 12.91260323 \\
0.339115677 & 4.814106788 & 0.160062054 & 10.19941358 \\
0.237068257 & 3.693860046 & 0.108985765 & 8.034966415 \\
0.165982548 & 2.82996896 & 0.074385824 & 6.314717425 \\
0.11637337 & 2.16511389 & 0.050880756 & 4.952001831 \\
0.081694823 & 1.65436022 & 0.034872078 & 3.875669903 \\
0.05741693 & 1.262626495 & 0.02394375 & 3.027768738 \\
\hline
\end{tabular}


Gráfico 10: Serie de datos Alcira. Intervalos de Predicción

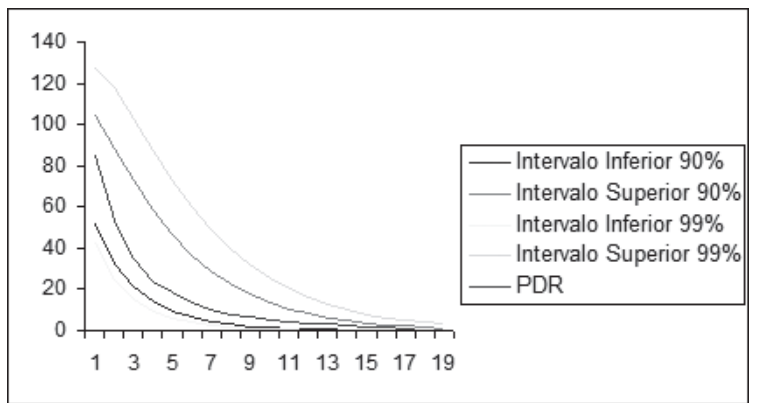

Tabla 12: Intervalos de predicción Serie de datos San Sebastián

\begin{tabular}{|c|c|c|c|}
\hline $\begin{array}{c}\text { Intervalo Inferior } \\
\mathbf{9 0 \%}\end{array}$ & $\begin{array}{c}\text { Intervalo Superior } \\
\mathbf{9 0 \%}\end{array}$ & $\begin{array}{c}\text { Intervalo Inferior } \\
\mathbf{9 9 \%}\end{array}$ & $\begin{array}{c}\text { Intervalo Superior } \\
\mathbf{9 9 \%}\end{array}$ \\
\hline 55.88673019 & 100.18978 & 47.3767122 & 118.1863187 \\
37.05699036 & 84.60462468 & 29.33655512 & 106.8698335 \\
25.27248782 & 69.46217465 & 18.98384242 & 92.47242596 \\
17.48837774 & 56.20551999 & 12.56787815 & 78.21076506 \\
12.21496518 & 45.05763431 & 8.442445384 & 65.191708 \\
8.588266561 & 35.88287462 & 5.730201016 & 53.78025856 \\
6.068676382 & 28.43353705 & 3.919925143 & 44.01970152 \\
4.305311079 & 22.44152956 & 2.698251813 & 35.80754227 \\
3.064237857 & 17.65495606 & 1.866771353 & 28.9799737 \\
2.186848251 & 13.85168449 & 1.297014671 & 23.35481062 \\
1.564300106 & 10.84259629 & 0.904425646 & 18.75342059 \\
1.121223944 & 8.470189423 & 0.632651602 & 15.01138882 \\
0.805060724 & 6.6052456 & 0.443764702 & 11.98298057 \\
0.578951589 & 5.142892942 & 0.31203377 & 9.542191672 \\
0.416928689 & 3.998711557 & 0.219886875 & 7.58197854 \\
0.300626698 & 3.105177536 & 0.155257439 & 6.012589635 \\
0.217014656 & 2.408557628 & 0.10982011 & 4.759531804 \\
0.156820624 & 1.866272483 & 0.077807254 & 3.761474669 \\
0.113431232 & 1.444700105 & 0.055208857 & 2.96825768 \\
0.082119271 & 1.117370989 & 0.039228088 & 2.339081415 \\
0.059499333 & 0.86350032 & 0.027908776 & 1.840915333 \\
0.043142831 & 0.666803832 & 0.019879272 & 1.447125689 \\
0.031304911 & 0.514548567 & 0.014175598 & 1.136311664 \\
0.022730227 & 0.39679592 & 0.01011888 & 0.891329981 \\
0.016514474 & 0.305800615 & 0.007230125 & 0.69848529 \\
\hline
\end{tabular}




\begin{tabular}{|c|c|c|c|}
\hline $\begin{array}{c}\text { Intervalo Inferior } \\
\mathbf{9 0 \%}\end{array}$ & $\begin{array}{c}\text { Intervalo Superior } \\
\mathbf{9 0 \%}\end{array}$ & $\begin{array}{c}\text { Intervalo Inferior } \\
\mathbf{9 9 \%}\end{array}$ & $\begin{array}{c}\text { Intervalo Superior } \\
\mathbf{9 9 \%}\end{array}$ \\
\hline 0.012005475 & 0.235535226 & 0.005170784 & 0.546863343 \\
0.00873239 & 0.181315212 & 0.003701191 & 0.427785311 \\
\hline
\end{tabular}

Gráfico 11: Serie de datos San Sebastián. Intervalos de Predicción

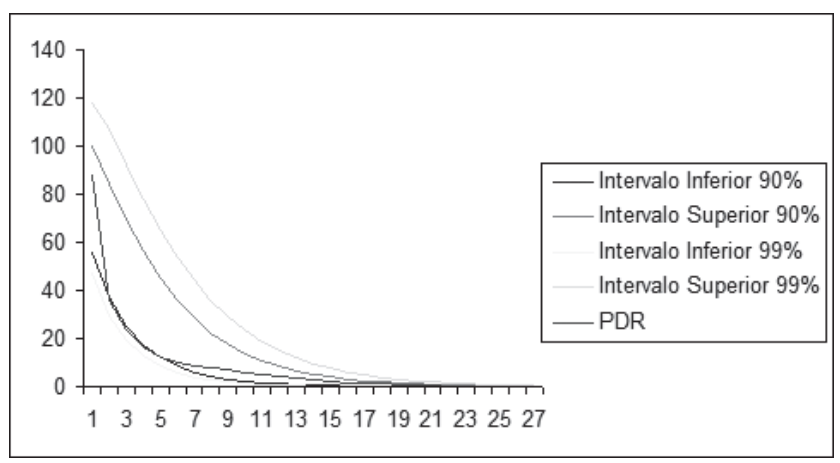

Gráfico 12: Serie de datos Barcelona. Intervalos de Predicción

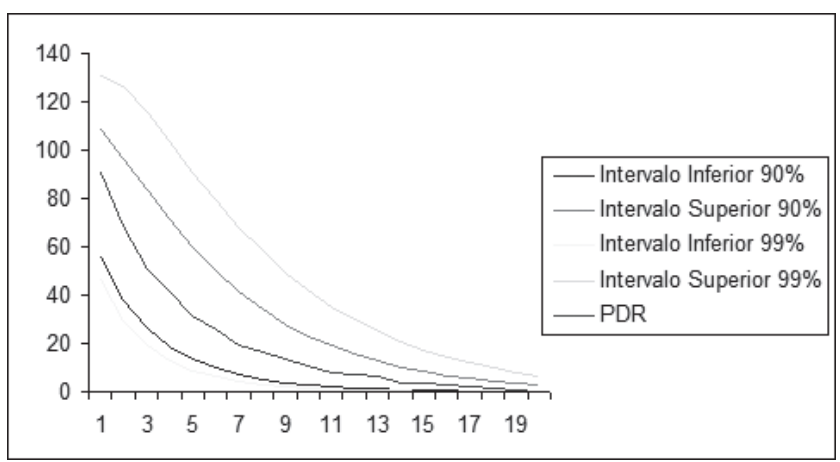

Gráfico 13 Serie de datos Zaragoza. Intervalos de Predicción

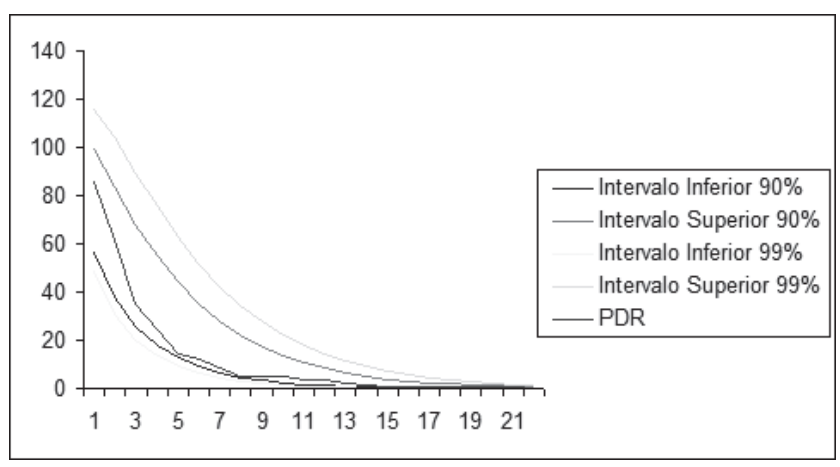


Gráfico 14: Serie de datos Valencia. Intervalos de Predicción

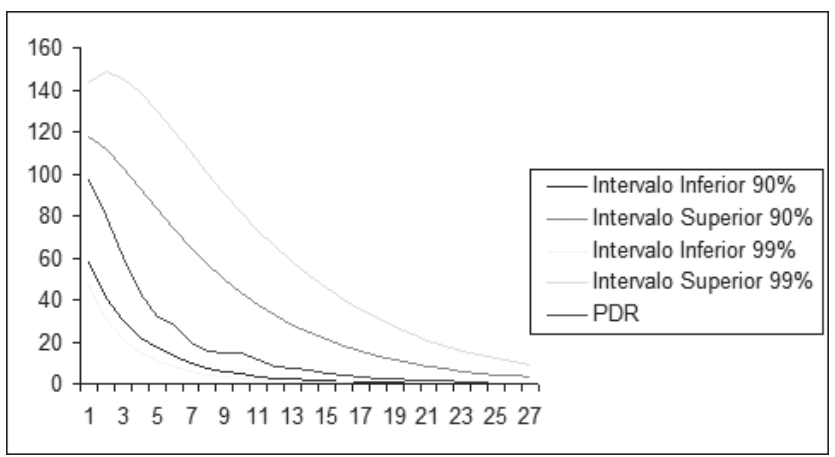

Gráfico 15: Serie de datos Murcia. Intervalos de Predicción

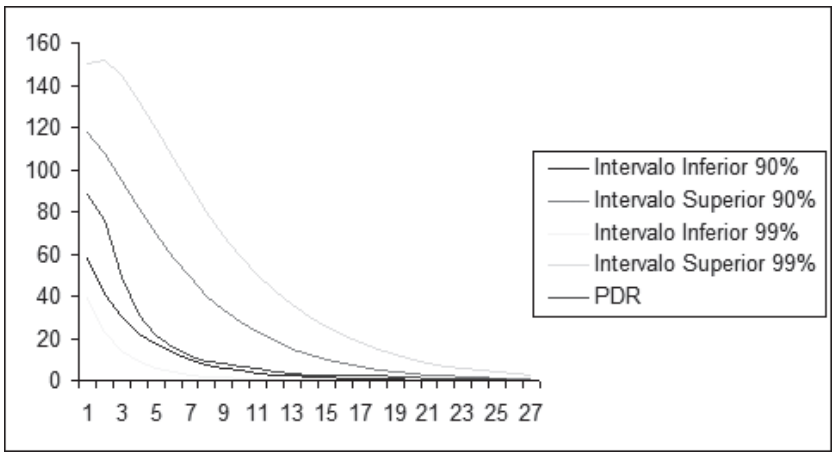

Los resultados obtenidos respecto a los intervalos de confianza muestran que no se mantiene la simetría respecto a la media debido a la transformación exponencial realizada. Además, los datos reales en las seis series consideradas están contenidos en los intervalos calculados, lo que permite concluir que la metodología de cálculo propuesta para las declaraciones de pérdidas catastróficas representa correctamente la realidad que se pretende modelar.

\section{CONCLUSIONES}

El modelo continúo propuesto por Pérez-Fructuoso $(2008,2009)$ se basa en definir una tasa de declaración como un valor constante, de manera que la cuantía de siniestros pendiente de declarar decrece exponencialmente, y el modelo exponencial que describe su dinámica viene caracterizado a través de la tasa que denominamos, tasa instantánea de declaración de siniestros. Dicha tasa funciona como un tanto instantáneo de descuento y tiene un efecto negativo en la ecuación diferencial estocástica representativa del modelo propuesto. Esto supone que, en promedio, el importe de las declaraciones de siniestros pendiente de declarar muestre, en el tiempo, un decrecimiento exponencial asintótico al eje de abscisas y, consecuentemente, 
la cuantía declarada de siniestros crezca asintóticamente a la cuantía total de la catástrofe. Esta tasa instantánea de declaración de siniestros constante, parámetro fundamental del modelo, se estima utilizando una metodología de Mínimos Cuadrados con Restricciones. Los valores obtenidos son muy similares a los de la tasa de declaración de siniestros estimada por Máxima Verosimilitud y la prueba JiCuadrado realizada no permite rechazar la hipótesis de distribución normal para la variable logaritmo neperiano de la cuantía de siniestros pendiente de declarar a partir de la cual calculamos el numerador de la ratio de siniestralidad objeto de estudio. A continuación, se lleva a cabo la estimación de la volatilidad del modelo aleatorio y el cálculo de los intervalos de confianza para los valores de la tasa de declaración de siniestros por el método de Mínimos Cuadrados con Restricciones. La estimación de la volatilidad incorporada por el proceso de Wiener en el modelo continuo aleatorio de evolución de las declaraciones de siniestros, ha sido realizada minimizando la suma de los errores al cuadrado y aplicando el resultado hallado para la estimación realizada sobre la tasa de declaración de siniestros por Mínimos Cuadrados con Restricciones. El contraste de los resultados obtenidos se realiza calculando los correspondientes intervalos de confianza, contraste del que se desprende que el modelo aleatorio describe de forma adecuada la realidad que pretendemos representar.

\section{REFERENCIAS BIBLIOGRÁFICAS}

- $\quad$ Arnold, L. (1974). Stochastic Differential Equations: Theory and Applications. John Wiley \& Sons, Inc.: New York, 1974.

- $\quad$ Cummins, J. D. y Geman, H. (1995). Pricing Catastrophe Insurance Futures and Call Spreads: An Arbitrage Approach. Journal of Fixed Income. 4 (4), 46-57.

Geman, H. y Yor, M. (1997). Stochastic time changes in catastrophe option pricing. Insurance: Mathematics and Economics. 21 (3), 185-193.

Johnson, N.L., S. Kotz, y N. Balakrishnan. Continuous univariate distributions, vol. 1. John Wiley \& Sons Inc.: New York, 1994.

Pérez-Fructuoso, M. J. y A. Alegre (2003). "Modelos de valoración de opciones sobre índices de catástrofes: análisis empírico y estimación de los parámetros del modelo alternativo". Anales del Instituto de Actuarios Españoles. Tercera Época, 9, pp. 121-151.

- Pérez-Fructuoso, M. J. (2008). Modeling loss index trigger for Cat bonds: A continuous Approach. Variance. 2 (2), 253-265.

- PÉrez-Fructuoso, M. J. (2009). Elaborating a catastrophic loss index for insurance-linked securities (ILS) a continuous model. Asian-Pacific Journal of Risk and Insurance. 3 (2), 34-45.

Nowak, P. y Romaniuk, M. (2013). Princing and simulations of catastrophe Bonds. Insurance: Mathematics and Economics. 52 (1), 18-28.

Van Den Boos, A. (2007). Parameter estimation for scientists and engineers. John Wiley \& Sons, Inc.: New Jersey. 